\section{A Rare Case of Massive Localized Lymphedema in a Morbidly Obese Patient}

Yeseul Moon, Jai-Kyong Pyon

Department of Plastic Surgery, Samsung Medical Center, Sungkyunkwan University School of Medicine, Seoul, Korea

Correspondence: Jai-Kyong Pyon

Department of Plastic Surgery, Samsung Medical Center, Sungkyunkwa University School of Medicine, 81 Irwon-ro, Gangnam-gu, Seoul 06351, Korea Tel: +82-2-3410-2235, Fax: +82-2-3410-0036

E-mail: pspriest.pyon@samsung.com

No potential conflict of interest relevant to this article was reported.

Received: 8 Apr 2015 • Revised: 26 May 2015• Accepted: 5 Jun 2015 pISSN: 2234-6163・ elSSN: 2234-6171

http://dx.doi.org/10.5999/aps.2016.43.1.125

Arch Plast Surg 2016:43:125-127

Copyright (C) 2016 The Korean Society of Plastic and Reconstructive Surgeons This is an Open Access article distributed under the terms of the Creative Commons Attribution Non-Commercial License (http://creativecommons.org/licenses/by-nc/3.0) which permits unrestricted non-commercial use, distribution, and reproduction in any medium, provided the original work is properly cited.

Massive localized lymphedema (MLL) is a rare condition that appears only in morbidly obese patients in the form of a large and painless lymphedema. MLL is caused by obstruction in the lymphatic channels resulting from obesity. Since it is a rare entity, no epidemiological study of its prevalence has been conducted to date. However, as the prevalence of obesity is increasing, it is expected that the incidence of MLL will also rise. It is therefore important for physicians to be familiar with this condition and its treatment. Here we present a case report of MLL.

A 54-year-old morbidly obese male was admitted to our hospital with a giant mass involving the lower abdomen and mons pubis. The mass had started in the abdominal area and gradually increased in size over six years and later obscured his penis and scrotum (Fig. 1). The patient's medical history included morbid obesity, hypertension, diabetes, gout, and a renal stone. There was no history of trauma or surgery in the abdominal area. On physical examination, the patient was morbidly obese, with a weight of $130 \mathrm{~kg}$, a height of $165 \mathrm{~cm}$, and a body mass index of $47.75 \mathrm{~kg} / \mathrm{m}^{2}$. The mass extended to the left knee when the patient stood at his full height. The skin overlying the mass had a 'peau d'orange' appearance. A CT scan of the patient showed skin and subcutaneous tissue thickening suggestive of lymphedema and a $3.3 \mathrm{~cm}$-diameter umbilical mesenteric fat hernia. We decided to combine abdominoplasty with liposuction and repair the abdominal wall. The incision line was designated along the bottom line of the mass lesion and the umbilicus. After the skin incision, dissection was performed down to the level of the rectus fascia, and further to the intersecting boundary of the xiphoid process and bilateral costal margins. This wide undermining allowed the greatest amount of inferior advancement of the abdominal flap (Fig. 2A). Once the abdominal flap was elevated, tumescent solution (normal saline $500 \mathrm{~mL}+2 \%$ lidocaine $12.5 \mathrm{~mL}+0.1 \%$
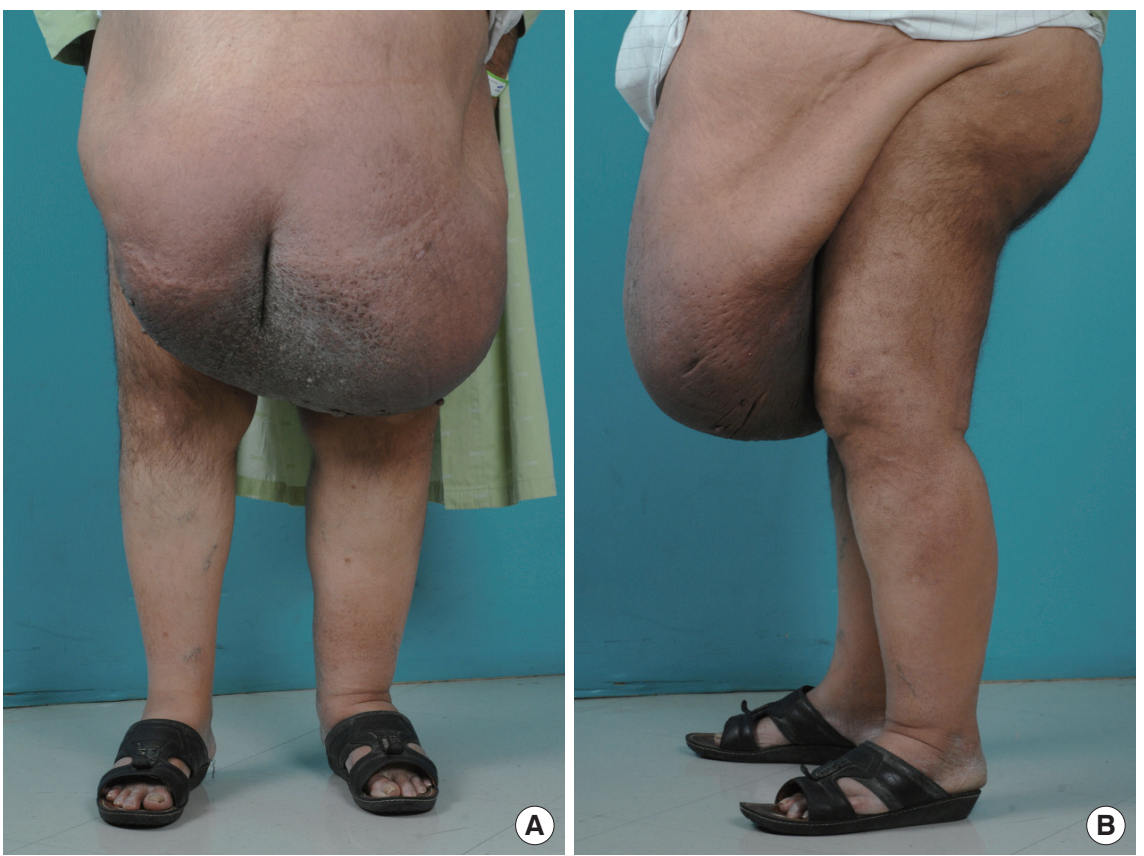

Fig. 1.

$(A, B)$ Preoperative photograph of a 54-year-old male with massive localized lymphedema at the abdomen. 
epinephrine $0.5 \mathrm{~mL}+8.4 \%$ sodium bicarbonate 12.5 $\mathrm{mL}$ ) was infiltrated on a subcutaneous plane of the abdominal flap. Five hundred $\mathrm{mL}$ of subcutaneous fat was removed from the abdominal flap using powerassisted liposuction. After liposuction, the upper flap was advanced inferiorly, where we closed the wound without much tension. After resection of the mass, a $1 \mathrm{~cm} \times 1 \mathrm{~cm}$ fascial defect was repaired. Two drains were inserted. The weight of the resected mass was $24.3 \mathrm{~kg}$ (Fig. 2B). We applied negative-pressure wound therapy (NPWT) to the closed incision. NPWT is traditionally indicated for open, uninfected wounds; however, more recently it has been used for incision management and has been shown to be effective for managing high-risk incisions related to orthopedic surgery, cardiac surgery, and abdominal wall reconstruction. The mechanism is speculated to be multifactorial and includes a soft-tissue splinting effect that resists lateral distracting forces and increased blood flow at the incision site [1]. After five days of NPWT, the incision wound was healthy and a simple dressing was applied. During the postoperative period, a large amount of fluid needed to be drained and there were a few small events such as minor wound dehiscence and seroma. We treated the wound dehiscence with secondary healing without any secondary procedure and the seroma was drained through percutaneous catheter. No clinical evidence of recurrence or other complications were shown three months after the surgery (Fig. 3).

MLL is a form of secondary lymphedema occurring as a result of impaired lymphatic drainage due to morbid obesity. It forms a large tumor mass, especially in the lower limb, hypogastrium, and mons pubis. The progressive growth can eventually result in an extremely large mass that hampers daily activities due to mobility limitation and also increases the risk of recurrent infections. The overlying skin often exhibits induration and a 'peau d'orange' appearance, consistent with chronic lymphedema. Its treatment depends highly on the location and severity of the mass. When possible, the conventional lymphedema treatment protocol of compression stockings, elevation of the lower body, frequent skin care, and observation is recommended to reduce symptoms and to reduce the incidence of wounds and infections. In more severe cases of wounds or infections, incision and drainage, debridement, or simple wedge resection may be performed. However, for most patients, in their later stages, complex surgical resection and reconstruction is often performed [2]. Although often benign, if left untreated MLL can progress to angiosarcoma, also known as StewartTreves syndrome [1]. The mortality associated with developing angiosarcoma is about 9\% [3]. Surgical resection improves the quality of life and is essential for functional rehabilitation. However, even after surgical treatment, the possibility of recurrence should not be overlooked. These considerations indicate the need for more research on MLL and its sequelae.

First described in 1998, MLL is a rare entity and is almost solely seen in morbidly obese patients.

Morbid obesity is still uncommon in Asian people, and only a few cases of MLL have been reported in Asia. This report is the first MLL case reported in Korea. However, with the increasing incidence of obesity, it is likely that clinicians will face more cases
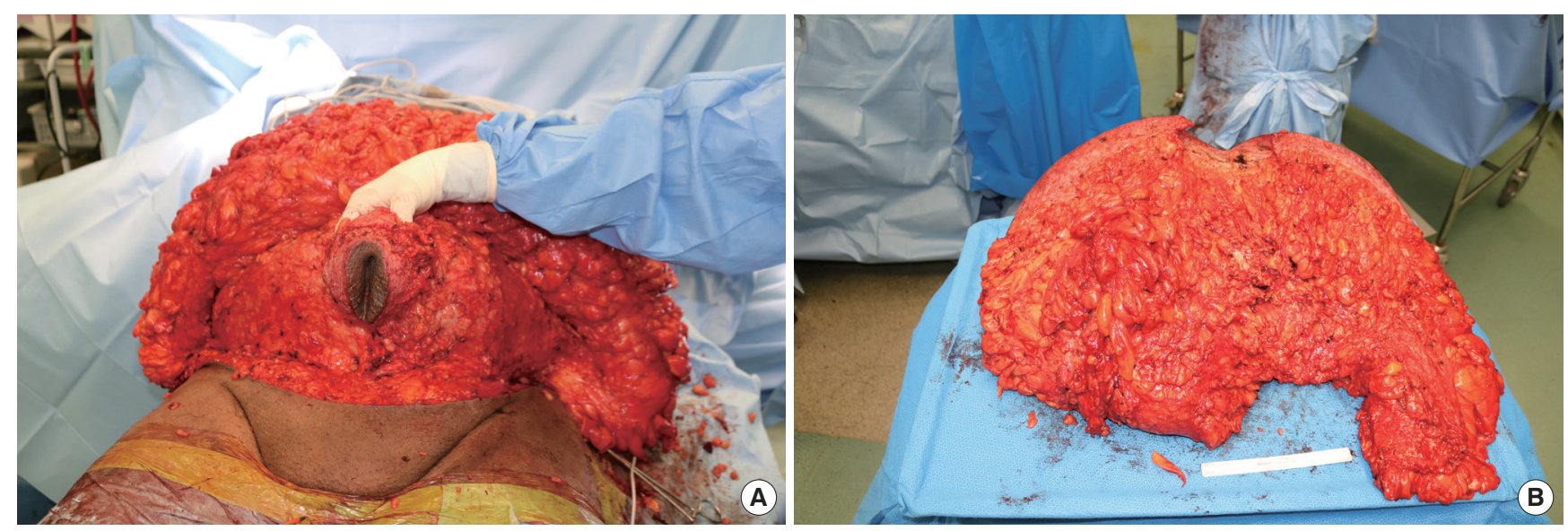

Fig. 2.

Intraoperative photograph. (A) The upper abdominal flap was harvested from the base of the lesion to the xiphoid process and costal margins.

(B) Removed specimen. 

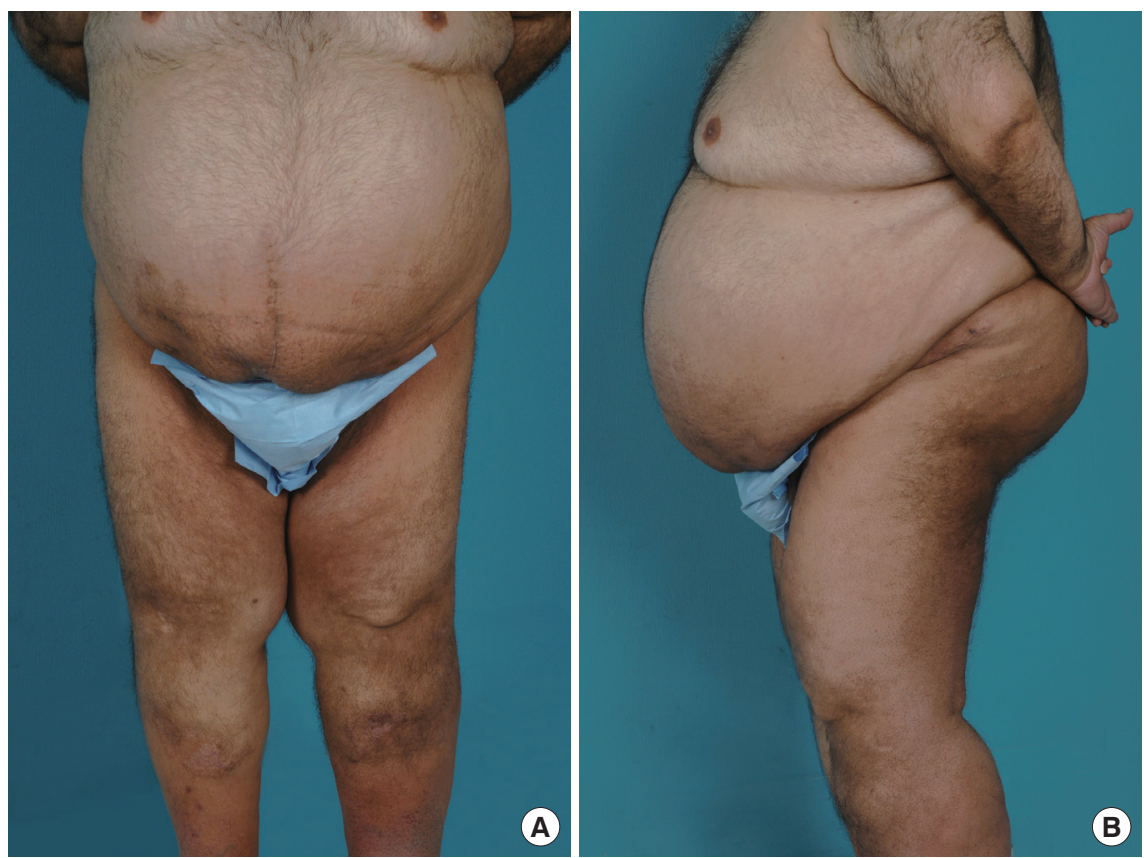

Fig. 3.

$(A, B)$ Three-month postoperative images showing no evidence of recurrence.

of MLL in the future. In this report, a rare case of MLL was successfully treated by surgical resection. Although there were minor complications such as wound dehiscence and seroma, there were no major complications or recurrence for a period of three months after surgery.

\section{References}

1. Chopra K, Tadisina KK, Brewer M, et al. Massive localized lymphedema revisited: a quickly rising complication of the obesity epidemic. Ann Plast Surg 2015;74:126-32.

2. Heller DS, Fitzhugh VA, Cracchiolo B, et al. Massive localized lymphedema of the vulva: report of a case and review of the literature.J Low Genit Tract Dis 2014;18:E46-9.

3. Fife C. Massive localized lymphedema, a disease unique to the morbidly obese: a case study. Ostomy Wound Manage 2014;60:30-5. 